\title{
HOMOGENIZATION OF A REFLECTING BARRIER BROWNIAN MOTION IN A CONTINUUM PERCOLATION CLUSTER IN $\boldsymbol{R}^{d}$
}

\author{
Dedicated to Professor Hiroshi Tanaka on his 60th birthday
}

\section{By HideKI TANEMURA*}

\section{Introduction and statement of result}

Let $\mathfrak{M}$ be the set of all countable subsets $\eta$ of $\boldsymbol{R}^{d}(d \geqq 2)$ satisfying $N_{K}(\eta)$ $<\infty$ for any compact subset $K$, where $N_{A}(\eta)$ is the number of points of $\eta$ in $A \subset \boldsymbol{R}^{d} . \mathfrak{M}$ is equipped with $\mathfrak{B}(\mathfrak{M})$ the $\sigma$-field which is generated by $\{\eta \in \mathfrak{M}$ : $\left.N_{A}(\eta)=n\right\}, A \in \mathfrak{B}\left(\boldsymbol{R}^{d}\right), n \in N$. For $\eta \in \mathfrak{M}, r>0$ and two disjoint regions $A_{1}$ and $A_{2}$ in $\boldsymbol{R}^{d}$, we say that a continuous curve $\gamma$ is an occupied (resp. a vacant) connection of $A_{1}$ and $A_{2}$ in a region $A$ with respect to $(\eta, r)$ if $\gamma \cap A_{1} \neq \emptyset, \gamma \cap A_{2}$ $\neq \emptyset, r \subset A$ and $\gamma \subset U_{r}(\eta)\left(r\right.$ esp. $\left.r \cap \overline{U_{r}(\eta)}=\emptyset\right)$, where $U_{r}(\eta)$ stands for the $r$-neighborhood of $\eta$ and $U_{r}(x)$ is the abbreviated form for $U_{r}(\{x\})$. A continuum percolation model is obtained if a distribution $\nu$ on the space $[0, \infty) \times \mathfrak{M}$ is given. In this paper we consider the case $\nu=\delta_{r} \otimes \mu_{\lambda}, r>0, \lambda>0$, where $\delta_{r}$ is the Dirac measure corresponding to the point $r$ and $\mu_{\lambda}$ is a Poisson distribution on $\mathfrak{M}$ with intensity measure $\lambda d x$, that is, for any disjoint system $\left\{A_{1}, A_{2}\right.$, $\left.\cdots, A_{m}\right\} \subset \mathfrak{B}\left(\boldsymbol{R}^{d}\right)$ such that $\left|A_{\imath}\right|=\int_{A_{\imath}} d x<\infty, i=1,2, \ldots, m, N_{A_{1}}(\eta), \ldots, N_{A_{m}}(\eta)$ are independent random variables on the probability space $\left(\mathfrak{M}, \mathfrak{B}(\mathfrak{M}), \mu_{\lambda}\right)$ and

$$
\mu_{\lambda}\left(N_{A_{\imath}}=n\right)=\frac{\left(\lambda\left|A_{\imath}\right|\right)^{n}}{n !} \exp \left(-\lambda\left|A_{\imath}\right|\right), \quad i=1,2, \ldots, m, n \in N \cup\{0\} .
$$

This percolation model is called the 'Poisson blob model'. (See Grimmett [9].) It should be viewed as a continuum analogue of the discrete site percolation model. Instead of sites being independently occupied we have a Poisson process on $\boldsymbol{R}^{d}$ with each Poisson point being the center of an occupied ball of radius $r$. Now we define two regions in $\boldsymbol{R}^{d}$,

* Research supported in part by Grant-in-Aid for Scientific Research (No. 04740102), Ministry of Education, Science and Culture.

Keywords: Poisson distribution, continuum percolation model, reflecting Brownian motion, central limit theorem.

Received January 27, 1993; revised September 28, 1993. 
$W(x, \eta, r)=\left\{y: \exists\right.$ an occupied connection of $\{x\}$ and $\{y\}$ in $\boldsymbol{R}^{d}$ w.r.t. $\left.(\eta, r)\right\}$, $W^{*}(x, \eta, r)=\left\{y: \exists\right.$ a vacant connection of $\{x\}$ and $\{y\}$ in $\boldsymbol{R}^{d}$ w.r.t. $\left.(\eta, r)\right\}$,

i. e., the occupied and vacant cluster of $x$, respectively. The critical values are defined as follows:

$$
\begin{aligned}
& \lambda_{c}(r)=\inf \left\{\lambda>0: \mu_{\lambda}(W(0, r) \text { is unbounded })>0\right\}, \\
& \lambda_{c}^{*}(r)=\sup \left\{\lambda>0: \mu_{\lambda}\left(W^{*}(0, r) \text { is unbounded }\right)>0\right\} .
\end{aligned}
$$

It was shown that $\lambda_{c}(r)=\lambda_{c}^{*}(r), r>0$ in the case where $d=2$ by Roy [16].

In this paper we study a homogenization problem of a reflecting barrier Brownian motion in an unbounded occupied (or vacant) cluster of the Poisson blob model.

Let $A$ be a Lipschitz domain in $\boldsymbol{R}^{d}$, that is, the boundary $\partial A$ of $A$ can be locally represented as the graph of a Lipschitz function defined on some open ball of $\boldsymbol{R}^{d-1}$. We define a Dirichlet form $\left(\mathcal{E}_{A}, H^{1}(A)\right)$ on $L^{2}(\bar{A}, d x)$ by

$$
\begin{aligned}
\mathcal{E}_{A}(u, v) & =\frac{1}{2} \int_{A} d x \nabla u(x) \cdot \nabla v(x), \quad u, v \in H^{1}(A), \\
H^{1}(A) & =\left\{u \in L^{2}(A, d x):|\nabla u| \in L^{2}(A, d x)\right\} .
\end{aligned}
$$

It is known that $\left\{\left.u\right|_{A}: u \in \boldsymbol{C}_{0}^{\infty}\left(\boldsymbol{R}^{d}\right)\right\}$ is dense in $H^{1}(A)$. (See Stein [17].) We introduce measurable subsets $\widehat{\mathfrak{M}}$ and $\mathfrak{M}_{x}, x \in \boldsymbol{R}^{d}$ of $\mathfrak{M}$ defined by

where

$$
\begin{gathered}
\widehat{\mathfrak{M}}=\{\eta \in \mathfrak{M} \backslash \Delta: W(0, \eta) \text { is unbounded }\}, \\
\mathfrak{M}_{x}=\{\eta \in \mathfrak{M} \backslash \Delta: W(x, \eta) \neq \emptyset\},
\end{gathered}
$$

$$
\Delta=\{\eta \in \mathfrak{M}:|x-y|=2 r \text { for some } x, y \in \eta\} \text {. }
$$

In case $\eta \in \mathfrak{M}_{x}, W(x, \eta)$ is a Lipschitz domain and $\left(\mathcal{E}_{W(x, \eta)}, H^{1}(W(x, \eta))\right)$ is a regular Dirichlet form on $\left.L^{2} \overline{(W(x, \eta)}, d x\right)$. Hence, by the theory of Dirichlet forms, we can construct the associated diffusion process starting from $x$, which is called a reflecting Brownian motion. (See Fukushima [7], [8], Bass and Hsu [1].) We denote by $\left(\Omega, \mathscr{F}, P_{x}^{\eta}, X(t)\right)$ this diffusion process if $\eta \in \mathfrak{M}_{x}$; otherwise $X(t)=x, t \geqq 0$. The process $X(t)$ is conservative and has a transition density $p^{\eta}(t, x, y)$. For a probability measure $\mu$ on $\mathfrak{M}$ with $\mu(\widehat{\mathfrak{M}})>0$ we put

$$
\hat{\mu}(d \eta)=\frac{1 \hat{\mathfrak{M}}(\eta)}{\mu(\widehat{\mathfrak{M}})} \mu(d \eta) .
$$

Changing the role of $W(x, \eta)$ into $W^{*}(x, \eta)$, we define $\widehat{\mathfrak{M}}^{*}, \mathfrak{M}_{x}^{*}, X^{*}(t), p^{n}(t, x, y)^{*}$ and $\hat{\mu}^{*}$ by the same way as $\hat{\mathfrak{M}}, \mathfrak{M}_{x}, X(t), p^{n}(t, x, y)$ and $\hat{\mu}$, respectively. For a probability measure $\mu$ on $\mathfrak{M}$ we put

$$
P^{\mu}=\int_{\mathfrak{M}} \mu(d \eta) P_{0}^{\eta}
$$


Our main result is the following theorem.

THEOREM 1. (i) If $\lambda>\lambda_{c}(r)$, then the process $\varepsilon X\left(t / \varepsilon^{2}\right)$ on $\left(\Omega, \mathscr{I}, P^{\mu_{\lambda \lambda}}\right)$ converges to $\hat{\sigma}(\lambda) B(t)$ in distribution on the space $\boldsymbol{C}\left([0, \infty) \rightarrow \boldsymbol{R}^{d}\right)$ equipped with the local uniform topology, where $B(t)$ is a d-dimensional Brownian motion and $\hat{\sigma}(\lambda)$ is a positive constant.

(ii) If $\lambda<\lambda_{c}^{*}(r)$, then the process $\varepsilon X^{*}\left(t / \varepsilon^{2}\right)$ on $\left(\Omega, \mathscr{I}, P^{\hat{\mu}}{ }_{\lambda}^{*}\right)$ converges to $\hat{\sigma}^{*}(\lambda) B(\lambda)$ in distribution on the space $\boldsymbol{C}\left([0, \infty) \rightarrow \boldsymbol{R}^{d}\right)$ equipped with the local uniform topology, where $\hat{\sigma}^{*}(\lambda)$ is a positive constant.

Central limit theorems for stochastic processes in random sets were studied by many authors. De Masi, Ferrari, Goldstein and Wick [5] proved a central limit theorem for a random walk in the Bernoulli percolation cluster. Tanemura [19] generalized their result to a jump type Markov process in a continuum percolation cluster. Bhattacharya [2] and Ochi [14] studied homogenization problems of reflecting barrier Brownian motions in the case of periodic domains and Osada [15] studied the problems in the case of domains with stationary scatterers under certain geometric conditions.

We prove the uniqueness of unbounded cluster of the continuum percolation model (Proposition 3.1). The unbounded cluster, say $W(\eta)$, is regarded as a domain with stationary scatterers. If $W(\eta)$ satisfied the geometric conditions in [15], Theorem 1 would be derived from [15]. However, $W(\eta)$ does not satisfy the conditions. The conditions were essentially used in [15] to prove the tightness and the nondegeneracy of the limit. Then we can obtain the convergence of finite dimensional distributions in the same way as [15] (Section 3).

The tightness of the rescaled diffusion processes is proved in Section 2 . Lyons and Zheng [13] obtained a decomposition of additive functionals of reversible Markov processes associated with Dirichlet forms. This decomposition is useful to check tightness. (See Takeda [18].) By virtue of the translation invariance of $\mu_{\lambda}$ we can apply their decomposition and show the tightness of the rescaled processes (Proposition 2.1). The technique used in Section 2 is thought to be applicable to more general situation.

We show the positivity of the diffusion constant $\hat{\sigma}(\lambda)$ in Section 4 . The positivity of the diffusion constant $\hat{\sigma}^{*}(\lambda)$ can be proved by the same way. We introduce jump type Markov processes $X^{n}(t), n \in N$ in the continuum percolation cluster. The diffusion constant $\hat{\sigma}(\lambda)$ is bounded from below by the inferior limit of the diffusion constants $\hat{\sigma}_{n}(\lambda)$ of $X^{n}(t), n \in N$. In the case of the random walk in the Bernoulli percolation cluster, the positivity of the diffusion constant was shown in [5] by using an estimate of the effective conductivity associated with this process. Their technique was generalized to jump type Markov processes in continuum percolation clusters in [19]. Then the key part for proving the positivity of $\hat{\sigma}(\lambda)$ is to estimate the effective conductivities associated with $X^{n}(t)$ uniformly in $n$ (Lemma 4.2). The proof of Lemma 4.2 is given in 
Section 5 by using a result about the continuum percolation model given in [20].

\section{Tightness}

In this section we establish the tightness of the rescaled processes $\varepsilon X\left(t / \varepsilon^{2}\right)$, $\varepsilon>0$.

PROPOSITION 2.1. Let $\mu$ be a translation invariant probability measure on $\mathfrak{M}$.

(i) If $\mu(\widehat{\mathfrak{M}})>0$, then $\left\{P^{\hat{\mu}} \circ\left(\varepsilon X\left(\cdot / \varepsilon^{2}\right)\right)^{-1}: \varepsilon \in(0,1)\right\}$ is tight in the space $\boldsymbol{C}([0, \infty)$ $\left.\rightarrow \boldsymbol{R}^{d}\right)$ equipped with the local uniform topology.

(ii) If $\mu\left(\widehat{\mathfrak{M}^{*}}\right)>0$, then $\left\{P^{\hat{*}} \circ\left(\varepsilon X^{*}\left(\cdot / \varepsilon^{2}\right)\right)^{-1}: \varepsilon \in(0,1)\right\}$ is tight in the space $\boldsymbol{C}\left([0, \infty) \rightarrow \boldsymbol{R}^{d}\right)$ equipped with the local uniform topology.

We give a proof of (i). A proof of (ii) is obtained similarly. For any $\delta>0$, put

$$
V_{h, T}=\left\{w \in \boldsymbol{C}\left([0, \infty) \rightarrow \boldsymbol{R}^{d}\right): \sup _{\substack{0 \leq s, t \leq T \\|t-s| \leqq h}}|w(t)-w(s)|>\delta\right\} .
$$

Then it is sufficient to show that

$$
\lim _{h \rightarrow 0} \sup _{\varepsilon \in(0,1)} P^{\mu} \circ\left(\varepsilon X\left(\frac{\cdot}{\varepsilon^{2}}\right)\right)^{-1}\left(V_{h, T}\right)=0, \quad \forall T>0 .
$$

Using the translation invariance of $\mu$, we obtain

$$
\begin{gathered}
P^{\mu} \circ\left(\varepsilon X\left(\frac{\dot{\bar{\varepsilon}}}{\varepsilon^{2}}\right)\right)^{-1}\left(V_{h, T}\right)=\int_{\mathfrak{M}} \mu(d \eta) P_{0}^{\eta_{\circ}}\left(\varepsilon X\left(\frac{\dot{\overline{2}}}{\varepsilon^{2}}\right)\right)^{-1}\left(V_{h, T}\right) \\
=\frac{1}{\left|U_{l}(0)\right|} \int_{U_{l}(0)} d y \int_{\mathfrak{M}} \mu(d \eta) P_{0}^{\tau-y} \eta \circ\left(\varepsilon X\left(\frac{\dot{\varepsilon^{2}}}{\varepsilon^{2}}\right)\right)^{-1}\left(V_{h, T}\right) \\
=\int_{\mathfrak{M}} \mu(d \eta) \frac{1}{\left|U_{l}(0)\right|} \int_{U_{r}(\eta) \cap U_{l}(0)} d y P_{y}^{\eta_{\circ}}\left(\varepsilon X\left(\frac{\dot{\varepsilon}}{\varepsilon^{2}}\right)\right)^{-1}\left(V_{h, T}\right),
\end{gathered}
$$

for any $l>0$, where $\tau_{x} \eta=\{x+y: y \in \eta\}$. Put $Q_{x}^{\eta, \varepsilon}=P_{x / \varepsilon^{\circ}}^{\circ}\left(\varepsilon X\left(\cdot / \varepsilon^{2}\right)\right)^{-1}$. Then

$$
P^{\mu} \circ\left(\varepsilon X\left(\frac{\cdot}{\varepsilon^{2}}\right)\right)^{-1}\left(V_{h, T}\right)=\int_{\mathfrak{M}} \mu(d \eta) \frac{1}{\left|U_{1}(0)\right|} \int_{\varepsilon U_{r}(\eta) \cap U_{1}(0)} d x Q_{x}^{\eta_{x} \varepsilon}\left(V_{h, T}\right),
$$

where $\alpha A=\{\alpha x: x \in A\}$ for $A \subset \boldsymbol{R}^{d}, \alpha>0$. Hence, Proposition 2.1 follows from the following lemma. We show the lemma by the same procedure as in Theorem 3.1 in Takeda [18].

LEMMA 2.1. For any $\eta \in \mathfrak{M}$

$$
\lim _{h \rightarrow 0} \sup _{\varepsilon \in(0,1)} \int_{\varepsilon U_{r}(\eta) \cap U_{1}(0)} d x Q_{x}^{\eta, \varepsilon}\left(V_{h, T}\right)=0, \quad \forall T>0 .
$$


Proof. From the definition of $X(t)$ this lemma is trivial if $\eta \in \Delta$. So we assume $\eta \in \mathfrak{M} \backslash \Delta$. Suppose that $x \in \varepsilon U_{r}(\eta)$. Then $\left(w(t), Q_{x}^{\eta_{1} \varepsilon}\right)$ is a diffusion process associated with the Dirichlet form $\left(\mathcal{E}_{\varepsilon U_{r}(\eta)}, H^{1}\left(\varepsilon U_{r}(\eta)\right)\right)$ starting from $x$. For any $\varepsilon \in(0,1)$ there exists an increasing sequence $\left\{a_{n}\right\}_{n \in N}$ such that $n+2<$ $a_{n} \leqq n+3$ and $A_{n}^{\varepsilon}[\eta]=\varepsilon U_{r}(\eta) \cap U_{a_{n}}(0)$ is a Lipschitz domain for any $n \in N$ with $A_{n}^{\varepsilon}[\eta] \neq \emptyset$. We denote the diffusion process starting from $x$ associated with the Dirichlet form $\left(\mathcal{E}_{A_{n}^{\varepsilon}[\eta]}, H^{1}\left(A_{n}^{\varepsilon}[\eta]\right)\right)$ by $\left(w(t), Q_{x}^{\eta_{1} \varepsilon, n}\right)$. Then,

$$
\begin{aligned}
& \int_{\varepsilon U_{r}(\eta) \cap U_{1}(0)} d x Q_{x}^{\eta_{x} \varepsilon}\left(V_{h, T}\right) \\
& \quad \leqq \int_{\varepsilon U_{r}(\eta) \cap U_{1}(0)} d x\left\{Q_{x}^{\eta_{,} \varepsilon, n}\left(\sup _{t \in[0, T]}|w(t)-w(0)|>n\right)+Q_{x}^{\eta, \varepsilon, n}\left(V_{h, T}\right)\right\} \\
& \leqq\left|A_{n}^{\varepsilon}[\eta]\right|\left\{Q^{\eta, \varepsilon, n}\left(\sup _{t \in[0, T]}|w(t)-w(0)|>n\right)+Q^{\eta, \varepsilon, n}\left(V_{h, T}\right)\right\},
\end{aligned}
$$

where

$$
Q^{\eta, \varepsilon, n}=\frac{1}{\left|A_{n}^{\varepsilon}[\eta]\right|} \int_{A_{n}^{\varepsilon}[\eta]} d x Q_{x}^{\eta, \varepsilon, n} .
$$

From the decomposition obtained by Lyons-Zheng [13] we claim

$$
w(t)-w(s)=\frac{1}{2}(M(t)-M(s))+\frac{1}{2}(\tilde{M}(T-t)-\tilde{M}(T-s)), \quad Q^{\eta, \varepsilon, n}-\mathbf{a} . \mathbf{s} .
$$

where $M(t)$ and $\tilde{M}(t)$ are $d$-dimensional Brownian motions with respect to $Q^{\eta, \varepsilon, n}$. Denoting by $P_{W}$ a Wiener measure on $\boldsymbol{C}\left([0, \infty) \rightarrow \boldsymbol{R}^{d}\right)$, by Doob's inequality we obtain

$$
\begin{aligned}
& Q^{\eta, \varepsilon, n}\left(\sup _{t \in[0, T]}|w(t)-w(0)|>n\right) \\
& \leqq Q^{\eta, \varepsilon, n}\left(\sup _{t \in[0, T]}|M(t)|>n\right) \\
& \quad+Q^{\eta, \varepsilon, n}\left(\sup _{t \in[0, T]}|\tilde{M}(T-t)-\tilde{M}(T)|>n\right) \\
& \leqq \\
& \quad \int_{C\left([0, \infty) \rightarrow R^{d}\right)} P_{W}(d w) \exp (|w(T)|) \exp (-n), \\
& Q^{\eta, \varepsilon, n}\left(V_{h, T} \leqq Q^{\eta, \varepsilon, n}\left(\sup _{\substack{0 \leq s, t \leq r \\
|t,-s| \leqq h}}|M(t)-M(s)|>\delta\right)\right. \\
& +Q^{\eta, \varepsilon, n}\left(\sup _{\substack{0 \leq s, t \leq T \\
|t-s| \leq h}}|\tilde{M}(t)-\tilde{M}(s)|>\delta\right) \\
& \leqq \frac{2 T}{h} P_{W}\left(\sup _{0 \leqq t \leqq 2 h}|w(t)|>\frac{\delta}{2}\right)
\end{aligned}
$$




$$
\begin{aligned}
& \leqq \frac{2 T}{h}\left(\frac{2}{\delta}\right)^{4} \int_{C\left([0, \infty) \rightarrow R^{d}\right)} P_{W}(d w)|w(2 h)|^{4} \\
& \leqq \frac{3 \cdot 2^{7} d T}{\delta^{4}} h .
\end{aligned}
$$

Setting $n=[-\log h]$ and combining (2.1), (2.3) and (2.4), we conclude that

$$
\begin{aligned}
& \int_{\varepsilon U_{r}(\eta) \cap U_{1}(0)} d x Q_{x}^{\eta, \varepsilon}\left(V_{h, T}\right) \\
& \quad \leqq\left|U_{1}(0)\right|\left\{2 \int_{C\left([0, \infty) \rightarrow R^{d}\right)} P_{W}(d w) \exp (|w(T)|)+\frac{3 \cdot 2^{7} d T}{\delta^{4}}\right\}(3-\log h)^{d} h,
\end{aligned}
$$

where $[\alpha]$ denotes the integer part of a nonnegative constant $\alpha$. Therefore, this completes the proof of Lemma 2.1.

We denote the expectation with respect to $P_{x}^{\eta}$ and $P^{\mu}$ by $E_{x}^{\eta}$ and $E^{\mu}$, respectively. We show a lemma which ensures the existence of the moment $E^{\hat{\mu}}\left[|X(t)|^{p}\right]$ (resp. $E^{\hat{\mu} *}\left[\left|X^{*}(t)\right|^{p}\right]$ ) for any $p \in N$ and any translation invariant probability measure $\mu$ on $\mathfrak{M}$ with $\mu(\widehat{\mathfrak{M}})>0$ (resp. $\mu\left(\widehat{\mathfrak{M}^{*}}\right)>0$ ).

LEMMA 2.2. Let $\mu$ be a translation invariant probability measure on $\mathfrak{M}$. For any $t \in(0, \infty)$ and any $l \in(0, \infty)$

$$
\begin{aligned}
& P^{\mu}\left(\sup _{t \in[0, T]}|X(t)| \geqq l\right) \leqq 2 P_{W}\left(\sup _{t \in[0, T]}|w(t)| \geqq l\right), \\
& P^{\mu}\left(\sup _{t \in[0, T]}\left|X^{*}(t)\right| \geqq l\right) \leqq 2 P_{W}\left(\sup _{t \in[0, T]}|w(t)| \geqq l\right),
\end{aligned}
$$

where $P_{W}$ is a Wiener measure on $\boldsymbol{C}\left([0, \infty) \rightarrow \boldsymbol{R}^{d}\right)$.

Proof. Let $\eta \notin \Delta$. There exists an increasing sequence $\left\{b_{n}\right\}_{n \in N}$ such that $(n+1) l<b_{n} \leqq(n+2) l$ and $A(n, \eta)=U_{r}(\eta) \cap U_{b_{n}}(0)$ is a Lipschitz domain for any $n \in \boldsymbol{N}$ with $A(n, \eta) \neq \emptyset$. We denote the diffusion process starting from $x \in$ $A(n, \eta)$ associated with the Dirichlet form $\left(\mathcal{E}_{\boldsymbol{A}(n, \eta)}, H^{1}(A(n, \eta))\right)$ by $\left(X(t), P_{x}^{\eta, n}\right)$ and put $P^{\eta, n}=(1 /|A(n, \eta)|) \int_{A(n, \eta)} d x P_{x}^{\eta, n}$.

$$
\begin{aligned}
& P^{\mu}\left(\sup _{t \in[0, T]}|X(t)| \geqq l\right)=\int_{\mathfrak{M}} \mu(d \eta) P_{0}^{\eta}\left(\sup _{t \in[0, T]}|X(t)| \geqq l\right) \\
& \quad=\int_{\mathfrak{M}} \mu(d \eta) \frac{1}{\left|U_{n l}(0)\right|} \int_{U_{n l}(0)} d x P_{0}^{\tau-x} \eta\left(\sup _{t \in[0, T]}|X(t)| \geqq l\right) \\
& \quad=\int_{\mathfrak{M}} \mu(d \eta) \frac{1}{\left|U_{n l}(0)\right|} \int_{U_{n l}(0)} d x P_{x}^{\eta}\left(\sup _{t \in[0, T]}|X(t)-X(0)| \geqq l\right)
\end{aligned}
$$




$$
\begin{aligned}
& \leqq \int_{\mathfrak{M} \backslash \Delta} \mu(d \eta) \frac{1}{\left|U_{n l}(0)\right|} \int_{A(n, \eta)} d x P_{x}^{\eta, n}\left(\sup _{t \in[0, T]}|X(t)-X(0)| \geqq l\right) \\
& \leqq\left(\frac{n+2}{n}\right)^{d} \int_{\mathfrak{M} \backslash \Delta} \mu(d \eta) P^{\eta, n}\left(\sup _{t \in[0, T]}|X(t)-X(0)| \geqq l\right)
\end{aligned}
$$

From the decomposition obtained by Lyons-Zheng [13] we claim

$$
X(t)-X(s)=\frac{1}{2}(N(t)-N(s))+\frac{1}{2}(\tilde{N}(T-t)-\tilde{N}(T-s)), \quad P^{\eta \cdot n}-\text { a.s. }
$$

where $N(t)$ and $\tilde{N}(t)$ are $d$-dimensional Brownian motions with respect to $P^{\eta, n}$. For this reason, we get

$$
\begin{aligned}
& P^{\eta, n}\left(\sup _{t \in[0, T]}|X(t)-X(0)| \geqq l\right) \\
& \quad \leqq P^{\eta, n}\left(\sup _{t \in[0, T]}|N(t)| \geqq l\right)+P^{\eta, n}\left(\sup _{t \in[0, T]}|\tilde{N}(T-t)-\tilde{N}(T)| \geqq l\right) \\
& =2 P_{W}\left(\sup _{t \in[0, T]}|w(t)| \geqq l\right),
\end{aligned}
$$

and conclude that

$$
P^{\mu}\left(\sup _{t \in[0, T]}|X(t)| \geqq l\right) \leqq 2\left(\frac{n+2}{n}\right)^{d} P_{W}\left(\sup _{t \in[0, T]}|w(t)| \geqq l\right), \quad \forall n \in N .
$$

This completes the proof of (i) of Lemma 2.2. We can prove (ii) by the same way.

\section{Convergence of finite dimensional distributions}

The purpose of this section is the following theorem.

THEOREM 3.1. (i) If $\mu_{\lambda}(\widehat{\mathfrak{M}})>0$, then the process $\varepsilon X\left(t / \varepsilon^{2}\right)$ on $\left(\Omega, \mathscr{F}, P^{\hat{\mu}} \lambda\right)$ converges to $\hat{\sigma}(\lambda) B(t)$ in the sense of finite dimensional distributions, where $\hat{\sigma}(\lambda)$ is a nonnegative constant determined by

$$
\hat{\sigma}(\lambda)^{2}=\lim _{t \rightarrow \infty} \frac{1}{t} E^{\hat{\mu}} \lambda\left[X_{1}(t)^{2}\right] .
$$

(ii) If $\mu_{\lambda}\left(\widehat{\mathfrak{M}^{*}}\right)>0$, then the process $\varepsilon X^{*}\left(t / \varepsilon^{2}\right)$ on $\left(\Omega, \mathscr{I}, P^{\hat{\mu}_{\lambda}^{*}}\right)$ converges to $\hat{\sigma}^{*}(\lambda) B(t)$ in the sense of finite dimensional distributions, where $\hat{\sigma}^{*}(\lambda)$ is a nonnegative constant determined by

$$
\hat{\sigma}^{*}(\lambda)^{2}=\lim _{t \rightarrow \infty} \frac{1}{t} E^{\hat{\mu}_{\lambda}^{*}\left[X_{1}^{*}(t)^{2}\right] .}
$$

Let $W(x, \eta)$ and $W^{*}(x, \eta)$ be the occupied cluster and the vacant cluster containing $x$ for $\eta$, which is defined in Section 1 . We denote the collections 
of all unbounded clusters for $\eta$ by $\mathscr{W}(\eta)$ and $\mathscr{W}^{*}(\eta)$ :

$$
\begin{aligned}
& \mathscr{W}(\eta)=\left\{\mathscr{W}(x, \eta): W(x, \eta) \text { is unbounded, } x \in \boldsymbol{R}^{d}\right\}, \\
& W^{*}(\eta)=\left\{W^{*}(x, \eta): W^{*}(x, \eta) \text { is unbounded, } x \in \boldsymbol{R}^{d}\right\} .
\end{aligned}
$$

We first study the number of elements of $\mathscr{W}(\eta)$ and $\mathscr{W}^{*}(\eta)$. Burton and Keane [3] proved the uniqueness of the infinite cluster of a site percolation model on $\boldsymbol{Z}^{d}$ under a translation invariant finite energy probability measure. Using their technique, we show the uniqueness of the unbounded cluster under a Poisson distribution $\mu_{\lambda}$.

PROPOSITION 3.1. (i) If $\mu_{\lambda}(\hat{\mathfrak{M}})>0$, then $\# \mathscr{W}(\eta)=1$ for $\mu_{\lambda}$-almost all $\eta$.

(ii) If $\mu_{\lambda}\left(\hat{\mathfrak{M}}^{*}\right)>0$, then $\# \mathscr{W}^{*}(\eta)=1$ for $\mu_{\lambda}$-almost all $\eta$.

Proof. First, note that $\# \mathscr{W}(\eta)$ is constant, $\mu_{\lambda}$-a.s. from the ergodicity of $\mu_{\lambda}$ under translation. Suppose that $\# \mathscr{W}(\eta)=n, \mu_{\lambda}$-a.s. $\eta, 2 \leqq n<\infty$. There exists $l_{0}>0$ such that

$$
\mu_{\lambda}\left(W \cap U_{l_{0}}(0) \neq \emptyset, \forall W \in \mathscr{W}\right)>0 .
$$

We define measurable subsets $\Lambda_{1}(l, \alpha), \Lambda_{2}(l, \alpha), l, \alpha>0$, of $\mathfrak{M}$ by

$$
\begin{aligned}
& \Lambda_{1}(l, \alpha)=\left\{\eta \in \mathfrak{M}: W \cap U_{l+\alpha}(0) \neq \emptyset, \forall W \in \mathscr{W}\left(\eta \backslash U_{l}(0)\right)\right\}, \\
& \Lambda_{2}(l, \alpha)=\left\{\eta \in \mathfrak{M}: U_{r}\left(\eta \cap U_{l}(0)\right) \supset U_{l+\alpha}(0)\right\} .
\end{aligned}
$$

Since any cluster $W$ is open set, from (3.1) we can choose $\alpha_{0} \in[0, r)$ such that $\mu_{\lambda}\left(\Lambda_{1}\left(l_{0}, \alpha_{0}\right)\right)>0$. It is easy to see that $\mu_{\lambda}\left(\Lambda_{2}\left(l_{0}, \alpha\right)\right)>0, \alpha \in[0, r)$. From a property of a Poisson distribution we have $\mu_{\lambda}\left(\Lambda_{1}\left(l_{0}, \alpha_{0}\right) \cap \Lambda_{2}\left(l_{0}, \alpha_{0}\right)\right)>0$. On the other hand, if $\eta \in \Lambda_{1}\left(l_{0}, \alpha_{0}\right) \cap \Lambda_{2}\left(l_{0}, \alpha_{0}\right)$, then $\# \mathscr{W}(\eta)=1$. Then, $\mu_{\lambda}(\# \mathscr{W}=1)>0$. It is a contradiction. Hence,

$$
\mu_{\lambda}(2 \leqq \# \mathscr{W}<\infty)=0 .
$$

Suppose that $\# \mathscr{W}(\eta)=\infty, \mu_{\lambda}$-a.s. $\eta$. We introduce the following notion. Let $l>0$. A point $x \in l Z^{d}$ is called an $l$-encounter site for $\eta \in \mathfrak{M}$, if $W(x, \eta)$ is unbounded and $W(x, \eta) \backslash \overline{U_{l / 2}(x)}$ has exactly three unbounded connected components. Denote by $\eta_{l}(\eta)$ the number of unbounded connected components of the open set $W(0, \eta) \backslash \overline{U_{l}(0)}$. We define a measurable subset $\Lambda_{3}(l, \alpha), l, \alpha>0$, of $\mathfrak{M}$ by

$$
\Lambda_{\mathbf{3}}(l, \alpha)=\left\{\eta \in \mathfrak{M}: \#\left\{W \in \mathscr{W}\left(\eta \backslash U_{l}(0)\right): W \cap U_{l+\alpha}(0) \neq \emptyset\right\} \geqq 3\right\} .
$$

By the same argument as the above we see that there exist $l_{1}>0$ and $\alpha_{1}>0$ such that $\mu_{\lambda}\left(\Lambda_{2}\left(l_{1}, \alpha_{1}\right) \cap \Lambda_{3}\left(l_{1}, \alpha_{1}\right)\right)>0$. If $\eta \in \Lambda_{2}\left(l_{1}, \alpha_{1}\right) \cap \Lambda_{3}\left(l_{1}, \alpha_{1}\right)$, then $\eta_{l_{1}+r}(\eta)$ $\geqq 3$. Then,

$$
\mu_{\lambda}\left(\Re_{l_{1}+r} \geqq 3\right)>0 \text {. }
$$


Since $\Re .(\eta)$ is a right continuous increasing function and satisfies for $\mu_{\lambda}$-a.s. $\eta$ $n_{l}(\eta)-\eta_{l_{-}}(\eta) \leqq 1$ for all $l>0$, there exists $l_{2}>0$ such that

$$
\mu_{\lambda}\left(\Re_{l_{2} / 2}=3\right)>0 \text {. }
$$

Then, we have

$$
\mu_{\lambda}\left(\left\{\eta: 0 \text { is an } l_{2} \text {-encounter site for } \eta\right\}\right)=\varepsilon>0 .
$$

It follows from the ergodic theorem that for almost all $\eta$

$$
\text { the number of } l_{2} \text {-encounter sites for } \eta \text { in } V_{k}=O\left(k^{d}\right), \quad k \rightarrow \infty \text {, }
$$

where $V_{k}=\left[-(k+1 / 2) l_{2},(k+1 / 2) l_{2}\right]^{d}, k \in N$. Put $\mathscr{W}(\eta)=\left\{W_{1}(\eta), W_{2}(\eta), \ldots\right\}$ and

$y_{i}(\eta)=\left\{Y: Y\right.$ is an unbounded connected component of $\left.W_{i}(\eta) \backslash V_{k}\right\}$.

Any $l_{2}$-encounter site $x$ for $\eta$ with $x \in W_{i}(\eta) \cap V_{k}$ determines a partition $\boldsymbol{P}(x)$ $=\left\{\boldsymbol{P}_{\mathbf{1}}(x), \boldsymbol{P}_{2}(x), \boldsymbol{P}_{3}(x)\right\}$ of $y_{i}(\eta)$. If partitions $\boldsymbol{P}(x)=\left\{\boldsymbol{P}_{1}(x), \boldsymbol{P}_{\mathbf{2}}(x), \boldsymbol{P}_{3}(x)\right\}$ and $\boldsymbol{P}(y)=\left\{\boldsymbol{P}_{1}(y), \boldsymbol{P}_{2}(y), \boldsymbol{P}_{3}(y)\right\}$ of $a_{i}(\eta)$ are determined by $l_{2}$-encounter sites $x, y \in$ $W_{i}(\eta) \cap V_{k}, x \neq y$, then $\boldsymbol{P}(x) \neq \boldsymbol{P}(y)$ and there is an ordering of each such that $\boldsymbol{P}_{1}(y) \supset \boldsymbol{P}_{1}(x) \cup \boldsymbol{P}_{2}(x)$. Using Lemma 2 in [3], if $\# \mathscr{P}_{\imath} \neq 0$, then

$$
\# \mathscr{Q}_{i}(\eta) \leqq \# \mathscr{y}_{i}(\eta)-2,
$$

where $\mathscr{Q}_{i}(\eta)$ is the collection of all partitions of $\mathscr{y}_{i}(\eta)$ determined by $l_{2}$-encounter sites for $\eta$ in $W_{i}(\eta) \cap V_{k}$. Since $\left|Y \cap\left(V_{k+\left(h / l_{2}\right)} \backslash V_{k}\right)\right| \geqq\left|U_{h}(0)\right|$ for any $Y \in q_{i}(\eta)$ with $\# \mathscr{Q}_{\imath} \neq 0$, we have

$$
\sum_{\imath \cdot \mathscr{P}_{i} \neq 0} \# \mathscr{y}_{i}(\eta)=O\left(k^{d-1}\right), \quad k \rightarrow \infty,
$$

and so

$$
\text { the number of } l_{2} \text {-encounter sites for } \eta \text { in } V_{k}=O\left(k^{d-1}\right), \quad k \rightarrow \infty \text {. }
$$

This contradicts (3.3). Hence, we have

$$
\mu_{\lambda}(\# \mathscr{W}=\infty)=0 .
$$

This completes the proof of (i). We can prove (ii) in the same way.

We introduce the environment process $\eta(t)$ (resp. $\eta^{*}(t)$ ) seen from $X(t)$ (resp. $\left.X^{*}(t)\right)$ defined by $\eta(t)=\tau_{-X(t)} \eta\left(\right.$ resp. $\left.\eta^{*}(t)=\tau_{-X *(t)} \eta\right)$. We denote by $O(\eta)$ (resp. $\left.O^{*}(\eta)\right)$ the union of all elements of $\mathscr{W}(\eta)\left(\right.$ resp. $\left.\mathscr{W}^{*}(\eta)\right)$. From Proposition 3.1, if $\mu_{\lambda}(\widehat{\mathfrak{M}})>0\left(\right.$ resp. $\left.\mu_{\lambda}\left(\widehat{\mathfrak{M}^{*}}\right)>0\right)$, then $O(\eta)\left(\right.$ resp. $\left.O^{*}(\eta)\right)$ is a connected open set for $\mu_{\lambda}$-almost all $\eta$. Then we can show the following by the same procedure as Theorem 2.1 in [15].

LNMMA 3.2. (i) Let $\mu_{\lambda}(\hat{\mathfrak{M}})>0$. Then the process $\left(\eta(t), P^{\hat{\mu}} \lambda\right)$ is an ergodic reversible Markov process with transition density 


$$
\boldsymbol{P}(t, \eta, \Lambda)= \begin{cases}P_{0}^{\eta}\left(X(t) \in \Lambda_{\eta}\right), & \text { if } \eta \in \mathfrak{M}_{0}, \\ \delta_{\eta}(\Lambda), & \text { otherwise, }\end{cases}
$$

$t>0, \eta \in \mathfrak{M}, \Lambda \subset \mathfrak{M}$, where $\Lambda_{\eta}=\left\{x \in \boldsymbol{R}^{d}: \tau_{-x} \eta \in \Lambda\right\}$.

(ii) Let $\mu_{\lambda}\left(\widehat{\mathfrak{M}^{*}}\right)>0$. Then the process $\left(\eta^{*}(t), P^{\hat{\mu}_{\lambda}^{*}}\right)$ is an ergodic reversible Markov process with transition density

$t>0, \eta \in \mathfrak{M}, \Lambda \subset \mathfrak{M}$.

$$
\boldsymbol{P}^{*}(t, \eta, \Lambda)= \begin{cases}P_{0}^{\eta}\left(X^{*}(t) \in \Lambda_{\eta}\right), & \text { if } \eta \in \mathfrak{M}_{0}^{*}, \\ \delta_{\eta}(\Lambda), & \text { otherwise, }\end{cases}
$$

Proof of Theorem 3.1. We introduce the measurable subset $\mathfrak{A}$ of $\boldsymbol{C}(\boldsymbol{R} \rightarrow \widehat{\mathfrak{M}})$ defined by

$$
\mathfrak{A}=\{\zeta \in \boldsymbol{C}(\boldsymbol{R} \rightarrow \mathfrak{M} \mathrm{\mathfrak {l }}): \exists \eta \in \widehat{\mathfrak{M}} \backslash \boldsymbol{\Xi} \text { s.t. } \boldsymbol{\zeta}(t) \in \Gamma(\eta), \forall t \geqq 0\},
$$

where $\Xi$ and $\Gamma(\eta)$ are measurable subsets of $\mathfrak{M}$ given by

$$
\begin{gathered}
\boldsymbol{E}=\left\{\eta \in \mathfrak{M}: \eta=\tau_{u} \eta \text { for some } u \in \boldsymbol{R}^{d} \backslash\{0\}\right\}, \\
\Gamma(\eta)=\left\{\tau_{-x} \eta: x \in \overline{W(0, \eta)}\right\}, \quad \eta \in \mathfrak{M} .
\end{gathered}
$$

We define a functional $F_{t}$ on $\mathfrak{A}$ by

$$
F_{t}(\zeta(\cdot))=x(t)-x(0), \quad t \in[0, \infty),
$$

where $x(\cdot) \in \boldsymbol{C}\left([0, \infty) \rightarrow \boldsymbol{R}^{d}\right)$ such that $\tau_{x(0)-x(t)} \zeta(0)=\zeta(t)$. The functional $F_{t}$ is additive and antisymmetric, that is,

$$
F_{t+s}(\zeta(\cdot))=F_{t}(\zeta(\cdot))+F_{s}(\zeta(t+\cdot)), \quad F_{t}(\zeta(\cdot))=-F_{t}(\zeta(t-\cdot)) .
$$

From the definition of the process $\eta(t)$ we have

$$
X(s)-X(0)=F_{s}(\eta(\cdot)), \quad P^{\hat{\mu}} \lambda \text {-a.s. }
$$

Then, $\left(X(t), P^{\hat{\mu}} \lambda\right)$ is a square integrable antisymmetric additive functional of the ergodic reversible Markov process $\left(\eta(t), P^{\hat{\mu}} \lambda\right)$. Applying Theorem 2.1 in [5], we obtain that the process $\varepsilon X\left(t / \varepsilon^{2}\right)$ converges to $D B(t)$ in the sense of finite dimensional distribution. (See Section 3 in [15].) Here $D$ is a symmetric and nonnegative definite $d \times d$ matrix determined by

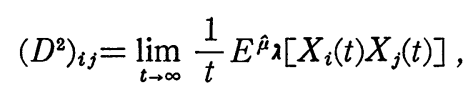

however, by the rotation invariance of $X(t)$ the matrix $D$ must be a constant multiple of the unit matrix. We have thus proved (i) of Theorem 3.1. We can prove (ii) in the same way. 


\section{Positivity of the diffusion constants}

Combining Proposition 2.1 and Theorem 3.1, we see that the rescaled processes $\varepsilon X\left(t / \varepsilon^{2}\right)$ and $\varepsilon X^{*}\left(t / \varepsilon^{2}\right)$ converge to $\hat{\sigma}(\lambda) B(t)$ and $\hat{\sigma}^{*}(\lambda) B(t)$, respectively. Then, the only assertion left to be proved is positivity of the diffusion constants $\hat{\sigma}(\lambda)$ and $\hat{\sigma}^{*}(\lambda)$. Let $\sigma(\lambda)$ and $\sigma^{*}(\lambda)$ be nonnegative constants determined by

$$
\sigma(\lambda)^{2}=\lim _{t \rightarrow \infty} \frac{1}{t} E^{\mu \lambda}\left[X_{1}(t)^{2}\right], \quad \sigma^{*}(\lambda)^{2}=\lim _{t \rightarrow \infty} \frac{1}{t} E^{\mu \lambda}\left[X_{1}^{*}(t)^{2}\right] .
$$

It is easy to see that $\hat{\sigma}(\lambda)^{2}=\left(\sigma(\lambda)^{2} / \mu_{\lambda}(\widehat{\mathfrak{M}})\right)$ and $\hat{\sigma}^{*}(\lambda)^{2}=\left(\sigma^{*}(\lambda)^{2} / \mu_{\lambda}\left(\widehat{\mathfrak{M}^{*}}\right)\right)$. Then, it is enough to show the following proposition.

Proposition 4.1. (i) If $\lambda>\lambda_{c}(r)$, then $\sigma(\lambda)>0$.

(ii) If $\lambda<\lambda_{c}^{*}(r)$, then $\sigma^{*}(\lambda)>0$.

For a subset $A$ of $\boldsymbol{R}^{d}$ and $\beta>0$, we define a measurable kernel $q^{\beta}[A]$ on $\boldsymbol{R}^{d} \times \mathscr{B}\left(\boldsymbol{R}^{d}\right)$ by

$$
q^{\beta}[A](x, d y)=\frac{\beta^{d+2} d}{\int v_{1}(0)|z|^{2} d z} \mathbf{1}_{[0,1 / \beta)}(|x-y|) \mathbf{1}_{A}(x) \mathbf{1}_{A}(y) d y .
$$

We abbreviate $q^{n}\left[U_{r}(\eta)\right]$ to $q_{\eta}^{n}$ for $\eta \in \mathfrak{M}, n \in N$. Denote by $\left(\Omega, \mathscr{T}, P_{x}^{\eta}, X^{n}(t)\right)$ a right continuous Markov process starting from $x$ with generator

$$
L_{\eta}^{n} u(x)=\int_{\boldsymbol{R}^{d}} q_{\eta}^{n}(x, d y)(u(y)-u(x)), \quad u \in \boldsymbol{C}_{\infty}\left(\boldsymbol{R}^{d}\right),
$$

where $\boldsymbol{C}_{\infty}\left(\boldsymbol{R}^{d}\right)$ is the space of continuous functions $u$ on $\boldsymbol{R}^{d}$ such that $u(x) \rightarrow 0$ as $|x| \rightarrow \infty$. Clearly $L_{\eta}^{n}$ can be regarded as a bounded linear operator on $L^{2}\left(\boldsymbol{R}^{d}, d x\right)$.

LEMMA 4.1. Let $\mu$ be a translation invariant probability measure on $\mathfrak{M}$. For any $t \in(0, \infty)$

$$
\lim _{n \rightarrow \infty} E^{\mu}\left[X_{1}^{n}(t)^{2}\right]=E^{\mu}\left[X_{1}(t)^{2}\right] .
$$

Proof. Let $\eta \in \mathfrak{M} \backslash \Delta$ and $W(x, \eta) \neq \emptyset$. Since $\mathcal{E}_{W(x, \eta)}$ is a Dirichlet form, by a standard result there is a nonpositive selfadjoint operator $L_{\eta}$ with $\mathscr{D}\left(\left(-L_{\eta}\right)^{1 / 2}\right)$ $=H^{1}(W(x, \eta))$ such that

Put

$$
\mathcal{E}_{W(x, \eta)}(u, v)=\left(\left(-L_{\eta}\right)^{1 / 2} u,\left(-L_{\eta}\right)^{1 / 2} v\right)_{L^{2}(W(x, \eta), d x)} .
$$

$$
\mathcal{A}=\left\{\left.u\right|_{W(x, \eta)}: u \in \boldsymbol{C}_{0}^{\infty}\left(\boldsymbol{R}^{d}\right), \frac{\partial u}{\partial \boldsymbol{n}}=0 \text { a. e. } d S \text { on } \partial W(x, \eta)\right\},
$$


where $S$ is the surface measure for $\partial W(x, \eta)$ and $n$ is the interior normal. We can show that $\mathcal{A}$ is a core for $L_{\eta}$. (See [7].) On the other hand it is easy to see that for any $u \in \mathcal{A}$

$$
\begin{gathered}
\lim _{n \rightarrow \infty} L_{\eta}^{n} u(y)=\Delta u(y)=L_{\eta} u(y), \quad \forall y \in W(x, \eta), \\
\sup _{n \in N} \sup _{y \in W(x, \eta)} L_{\eta}^{n} u(y)<\infty,
\end{gathered}
$$

and so

$$
L_{\eta}^{n} u \longrightarrow L_{\eta} u, \quad \text { as } n \rightarrow \infty \text { in } L^{2}(W(x, \eta), d x) .
$$

Applying Theorem 5.2 in Trotter [21], we obtain that for any $u \in L^{2}(W(x, \eta), d x)$ and $t \in(0, \infty)$

$$
T_{\eta}^{n}(t) u \longrightarrow T_{\eta}(t) u \quad \text { as } n \rightarrow \infty \text { in } L^{2}(W(x, \eta), d x),
$$

where $T_{\eta}^{n}(t)$ and $T_{\eta}(t)$ are semigroups on $L^{2}(W(x, \eta), d x)$ associated with $L_{n}^{n}$ and $L^{\eta}$, respectively. Hence, for any $u, v \in L^{2}\left(\boldsymbol{R}^{d}, d x\right)$

$$
\lim _{n \rightarrow \infty} \int_{W(x, \eta)} d y E_{y}^{\eta}\left[u\left(X^{n}(0)\right) v\left(X^{n}(t)\right)\right]=\int_{W(x, \eta)} d y E_{y}^{\eta}[u(X(0)) v(X(t))] .
$$

Noting that $X^{n}(t)=X(t)=y, t \in[0, \infty), P_{y}^{\eta}$-a.s. if $y \notin U_{r}(\eta)$, we obtain

$$
\lim _{n \rightarrow \infty} \int_{U_{1}(0)} d y E_{y}^{\eta}\left[u\left(X^{n}(0)\right) v\left(X^{n}(t)\right)\right]=\int_{U_{1}(0)} d y E_{y}^{\eta}[u(X(0)) v(X(t))] .
$$

From the translation invariance of $\mu$ we have

$$
\begin{aligned}
E^{\mu} & {\left[u\left(X^{n}(t)\right)\right]=\int_{\mathfrak{M}} \mu(d \eta) E_{0}^{\eta}\left[u\left(X^{n}(t)\right)\right] } \\
& =\frac{1}{\left|U_{1}(0)\right|} \int_{U_{1}(0)} d x \int_{\mathfrak{M}} \mu(d \eta) E_{x}^{\tau} x^{\eta}\left[u\left(X^{n}(t)-X^{n}(0)\right)\right] \\
& =\int_{\mathfrak{M}} \mu(d \eta) \frac{1}{\left|U_{1}(0)\right|} \int_{U_{1}(0)} d x E_{x}^{\eta}\left[u\left(X^{n}(t)-X^{n}(0)\right)\right] .
\end{aligned}
$$

Similarly,

$$
E^{\mu}[u(X(t))]=\int_{\mathfrak{M}} \mu(d \eta) \frac{1}{\left|U_{1}(0)\right|} \int_{U_{1}(0)} d x E_{x}^{\eta}[u(X(t)-X(0))] .
$$

Hence, from (4.1), (4.2) and (4.3) we arrive at

$$
\lim _{n \rightarrow \infty} E^{\mu}\left[u\left(X^{n}(t)\right)\right]=E^{\mu}[u(X(t))],
$$

for any $u \in \boldsymbol{C}_{0}\left(\boldsymbol{R}^{d}\right)$ and $t \in(0, \infty)$. Then for the proof of the lemma it is sufficient to show that

$$
\lim _{M \rightarrow \infty} \sup _{n \in N} E^{\mu}\left[X_{1}^{n}(t)^{2}: X_{1}^{n}(t)^{2}>M\right]=0
$$


This equality is derived from the estimate

$$
\sup _{n \in N} E^{\mu}\left[\left|X_{1}^{n}(t)\right|^{3}\right]<\infty,
$$

which can be shown by the same argument as in the proof of Lemma 2.2 .

We give the definition of an electrical network and an effective conductivity in the context of an electrical network, which is a generalization of that in Doyle and Snell [6]. Let $(q, m)$ be a pair of a measurable kernel $q$ on $\boldsymbol{R}^{d} \times$ $\mathscr{B}\left(\boldsymbol{R}^{d}\right)$ and a Radon measure $m$ on $\boldsymbol{R}^{d}$ satisfying

$$
q(x, d y) m(d x)=q(y, d x) m(d y) .
$$

We call the pair $(q, m)$ an electrical network. Throughout this paper, we consider the case where $q(\cdot, A)$ is bounded for any bounded set $A$. For $k \in N$, the effective conductivity in $(q, m)$ is defined by

$$
g_{k}(q, m)=\min _{u}\left\{\frac{1}{2} \int_{[-k-1 / 2, k+1 / 2) d} m(d x) \int_{[-k-1 / 2, k+1 / 2) d} q(x, d y)(u(y)-u(x))^{2}\right\},
$$

where $u$ ranges all measurable functions $u$ on $[-k-1 / 2, k+1 / 2)^{d}$ satisfying $u=0$ on $[-k-1 / 2,-k+1 / 2) \times[-k-1 / 2, k+1 / 2)^{d-1}$ and $u=1$ on $[k-1 / 2, k+1 / 2)$ $\times[-k-1 / 2, k+1 / 2)^{d-1}$.

We state the following result whose proof is given in Section 5 .

LEMMA 4.2. Let $\lambda>\lambda_{c}(r)$. Then there exists a positive constant $c(\lambda)$ such that

$$
\varliminf_{k \rightarrow \infty}(2 k)^{2-d} \mathcal{g}_{k}\left(q_{\eta}^{n}, d x\right) \geqq c(\lambda), \quad \mu_{\lambda}-a . s . \quad \forall n \in N .
$$

Now, we are in a position to prove Proposition 4.1.

Proof of Proposition 4.1. In [19] we have shown that $X^{n}(t)$ is an antisymmetric additive functional of a reversible Markov process. By the same calculation as in the proof of Theorem 2.2 in [5], we obtain that

$$
\begin{aligned}
E^{\mu \lambda}\left[X_{1}^{n}(t)^{2}\right]= & t \int_{\mathfrak{M}} \mu_{\lambda}(d \eta) \int_{R^{d}} q_{\eta}^{n}(0, d u) u_{1}^{2} \\
& -2 \int_{0}^{t} d s \int_{0}^{s} d v \int_{\mathfrak{M}} \mu_{\lambda}(d \eta) G(\eta) S_{v} G(\eta),
\end{aligned}
$$

where $S_{t}$ is the semigroup on $L^{2}\left(\mathfrak{M}, \mu_{\lambda}\right)$ associated with the reversible process and $G(\eta)=\int_{R d} q_{\eta}^{n}(0, d u) u_{1}$. Since $S_{t}$ is symmetric, we see that

$$
\lim _{t \rightarrow \infty} \frac{1}{t} E^{\mu_{\lambda}}\left[X_{1}^{n}(t)^{2}\right] \leqq \frac{1}{t} E^{\mu \lambda}\left[X_{1}^{n}(t)^{2}\right], \quad \forall t \in(0, \infty) .
$$

On the other hand, we proved in [19] that 


$$
\lim _{t \rightarrow \infty} \frac{1}{t} E^{\mu_{\lambda}}\left[X_{1}^{n}(t)^{2}\right] \geqq \int_{\mathfrak{m}} \mu_{\lambda}(d \eta) \varliminf_{k \rightarrow \infty} 2(2 k)^{2-d} g_{k}\left(q_{\eta}^{n}, d x\right) .
$$

Then, from Lemmas 4.1 and 4.2 we conclude that

$$
\begin{aligned}
\sigma(\lambda)^{2} & =\lim _{t \rightarrow \infty} \frac{1}{t} E^{\mu_{\lambda}}\left[X_{1}(t)^{2}\right] \\
& =\lim _{t \rightarrow \infty} \frac{1}{t} \lim _{n \rightarrow \infty} E^{\mu_{\lambda}}\left[X_{1}^{n}(t)^{2}\right] \\
& \geqq \lim _{n \rightarrow \infty} \lim _{t \rightarrow \infty} \frac{1}{t} E^{\mu_{\lambda}}\left[X_{1}^{n}(t)^{2}\right] \\
& \geqq \lim _{n \rightarrow \infty} \int_{\mathfrak{M}} \mu_{\lambda}(d \eta) \varliminf_{k \rightarrow \infty} 2(2 k)^{2-d} g_{k}\left(q_{\eta}^{n}, d x\right) \\
& \geqq 2 c(\lambda) .
\end{aligned}
$$

This completes the proof of (i). A proof of (ii) is obtained similarly.

\section{Proof of Lemma 4.2}

In this section we give the proof of Lemma 4.2. First we introduce a site percolation model on $\boldsymbol{Z}^{d}$. For each $x=\left(x_{1}, x_{2}, \ldots, x_{d}\right)$ and $y=\left(y_{1}, y_{2}, \ldots, y_{d}\right)$ we write $\langle x, y\rangle$ and say that $x$ is adjacent to $y$ if $\sum_{j=1}^{d}\left|x_{j}-y_{j}\right|=1$. The element $x \in \boldsymbol{Z}^{d}$ is called a site. Put $\boldsymbol{X}=\{0,1\}^{\boldsymbol{Z}^{d}}$ and equip $\boldsymbol{X}$ with the $\sigma$-field generated by $\{\xi \in \boldsymbol{X}: \xi(x)=0\}, x \in \boldsymbol{Z}^{d}$. For $\xi \in \boldsymbol{X}$, a site $x \in \boldsymbol{Z}^{d}$ is called occupied (resp. vacant), if $\xi(x)=1$ (resp. $\xi(x)=0$ ). We write $x \leftrightarrow y$ if there is an occupied path from $x$ to $y$; there is a sequence $x^{0}=x, x^{1}, \ldots, x^{n}=y$ of occupied sites so that $\left\langle x^{m-1}, x^{m}\right\rangle, 1 \leqq m \leqq n$. For $A_{1}, A_{2} \subset Z^{d}$ we write $A_{1} \leftrightarrow A_{2}$ if there are sites $x \in A_{1}, y \in A_{2}$ such that $x \leftrightarrow y$. We define a region in $\boldsymbol{Z}^{d}$ by

$$
C(x, \xi)=\left\{y \in Z^{d}: x \leftrightarrow y\right\},
$$

i. e., the occupied cluster containing the site $x$. For a given $l \in N$ define a measurable function $\pi_{l}: \mathfrak{M} \rightarrow \boldsymbol{X}$ by

$$
\pi_{l}(\eta)(x)= \begin{cases}1 & \text { if } D_{l}(x) \cap \eta \neq \emptyset, \\ 0 & \text { if } D_{l}(x) \cap \eta=\emptyset,\end{cases}
$$

$\eta \in \mathfrak{M}, x \in \boldsymbol{Z}^{d}$, where $D_{l}(x)$ denotes the union of the cubes

$$
I(y)=\prod_{i=1}^{d}\left[y_{i}-\frac{1}{2}, y_{i}+\frac{1}{2}\right)
$$

with $y \in Z^{d}$ and $I(y) \subset U_{l}(x)$. Define a probability measure $P_{p}$ on $\boldsymbol{X}$ by $P_{p}=$ $\mu_{\theta^{-1}(p)} \pi_{l}^{-1}$, where $\theta(\lambda)=1-\exp (-\lambda)$ and $\theta^{-1}$ is the inverse function of $\theta$. The 
critical value is defined as follows:

$$
p_{c}=p_{c}(l)=\inf \left\{p>0: P_{p}(\# C(0)=\infty)>0\right\} \text {. }
$$

For $\xi \in X$ we denote by $\Re_{k}(\xi)$ the maximal number of disjoint occupied paths in a cube $\{-k,-k+1, \ldots, k\}^{d}$ from $\{-k\} \times\{-k,-k+1, \ldots, k\}^{d-1}$ to $\{k\} \times$ $\{-k,-k+1, \ldots, k\}^{d-1}$. In Tanemura [20] we obtain the following proposition which is an analogue of the result shown by Grimmett and Marstrand [11] in the case of the Bernoulli percolation model.

Proposition 5.1. If $p>p_{c}$, then there exist $c_{1}(p), c_{2}(p)>0$ such that

$$
P_{p}\left(\Re_{k} \geqq c_{1}(p) k^{d-1}\right) \geqq 1-\exp \left(-c_{2}(p) k^{d-1}\right), \quad \forall k \in N .
$$

Put ${\hat{g_{k}}}_{k}(q, m)=(2 k)^{2-d} g_{k}(q, m)$ and $\hat{\Re}_{k}(\xi)=k^{1-d} \Re_{k}(\xi)$ and introduce the nonnegative constant $c_{3}(\beta, c)$ defined by

$$
c_{3}(\beta, c)=\inf \left\{\widehat{g}_{k}\left(q^{\beta}[I[\xi]], d x\right): k \in N, \xi \in X \text { with } \widehat{\Re}_{k}(\xi) \geqq c\right\},
$$

for $\beta>0$ and $c>0$, where $I[\xi]=\bigcup_{\xi(x)=1} I(x)$.

LeMmA 5.1. Suppose that $\beta \in\left(0,1 / \sqrt{d+3)}\right.$ and $c>0$. Then, $c_{3}(\beta, c)>0$.

Proof. Let $\left(\tilde{q}_{\xi}, \tilde{\boldsymbol{m}}\right), \boldsymbol{\xi} \in \boldsymbol{X}$ be the electrical network defined by

$$
\tilde{q}_{\xi}(x, d y)=\mathbf{1}_{\boldsymbol{Z}}(x) \xi(x) \sum_{\substack{u \in Z^{d} \\\langle u, x\rangle}} \boldsymbol{\xi}(u) \delta_{u}(d y), \quad \tilde{m}(d x)=\sum_{u \in Z^{d}} \delta_{u}(d x) .
$$

It was already shown that for any $c>0$

$$
c_{4}(c)=\inf \left\{\widehat{g_{k}}\left(\tilde{q}_{\xi}, \tilde{m}\right): k \in N, \xi \in X \text { with } \widehat{\eta_{k}}(\xi) \geqq c\right\}>0 .
$$

(See Section 6 in Grimmett and Kesten [10] or Section 3 in Chayes and Chayes [4].) Put

$$
\hat{q}_{\xi}(x, d y)=\sum_{u, v \in Z^{d}} \tilde{q}_{\xi}(u,\{v\}) \mathbf{1}_{I(u)}(x) \mathbf{1}_{I(v)}(y) d y .
$$

Then, we have

$$
\hat{q}_{\xi}(x, d y) \leqq c_{5}(\beta) q^{\beta}[I[\xi]](x, d y), \quad \text { if } \beta \in\left(0, \frac{1}{\sqrt{d+3}}\right),
$$

where $c_{5}(\beta)=\beta^{-d-2} d \int_{U_{1}(0)}|z|^{2} d z$.

It is easy to see that for any $\xi \in X$

$$
\mathcal{I}_{k}\left(\tilde{q}_{\xi}, \tilde{m}\right)=\mathcal{I}_{k}\left(\hat{q}_{\xi}, d x\right), \quad \forall k \in N,
$$

and 


$$
\mathcal{I}_{k}\left(\hat{q}_{\xi}, d x\right) \leqq c_{5}(\beta) \mathcal{I}_{k}\left(q^{\beta}[I[\xi]], d x\right), \quad \forall k \in N, \quad \forall \beta \in\left(0, \frac{1}{\sqrt{d+3}}\right),
$$

from (5.3). Combining (5.4) and (5.5), we obtain Lemma 5.1 from (5.2).

Proof of Lemma 4.2. Note that $\left(\mu_{\lambda}, \eta\right)=\left(\mu_{\alpha^{d} \lambda}, \alpha \eta\right)$ for any $\alpha>0$. Then, we see that $\lambda_{c}(r)=\alpha^{d} \lambda_{c}(\alpha r), \alpha>0$. Let $\lambda>\lambda_{c}(r)$. Choose $\delta_{0}>0$ such that $\lambda>$ $\left(r /\left(r-\delta_{0}\right)\right)^{d} \lambda_{c}(r)=\lambda_{c}\left(r-\delta_{0}\right)$. We first show that there exist $\alpha, l \in N$ with $\alpha>$ $\sqrt{d+3}$ satisfying

$$
U_{r-\delta_{0}}(\eta) \subset \frac{1}{n \alpha} I\left[\pi_{n l}(n \alpha \eta)\right] \subset U_{r}(\eta), \quad \forall \eta \in \mathfrak{M}, \quad \forall n \in N .
$$

From the definition we have $U_{l-\sqrt{a}}(x) \subset D_{l}(x) \subset U_{l}(x)$ for any $x \in \boldsymbol{R}^{d}$. Then

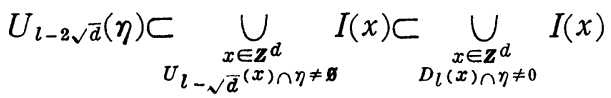

$$
\begin{aligned}
& =I\left[\pi_{l} \eta\right] \subset \bigcup_{\substack{x \in Z^{d} \\
U_{l}(x) \cap \eta \neq \emptyset}} I(x) \subset U_{l+\sqrt{\alpha}}(\eta),
\end{aligned}
$$

for any $l \in N$ and $\eta \in \mathfrak{M}$. Hence

$$
\begin{aligned}
U_{(l / \alpha)-(2 \sqrt{d} / n \alpha)}(\eta) & =\frac{1}{n \alpha} U_{n l-2 \sqrt{d}}(n \alpha \eta) \subset \frac{1}{n \alpha} I\left[\pi_{n l}(n \alpha \eta)\right] \\
\subset \frac{1}{n \alpha} U_{n l+\sqrt{d}}(n \alpha \eta) & =U_{(l / \alpha)+(\sqrt{d} / n \alpha)}(\eta) .
\end{aligned}
$$

Choosing $l$ and $\alpha$ such that $r-\delta_{0}<(1 / \alpha)(l-2 \sqrt{d})<(1 / \alpha)(l+\sqrt{d})<r$, we obtain (5.6).

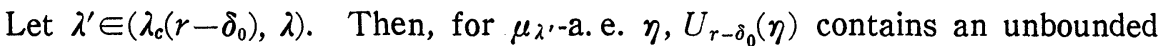
connected component and so $I\left[\pi_{l}(\alpha \eta)\right]$ contains an unbounded connected component. Hence, $I\left[\pi_{l}(\eta)\right]$ contains an unbounded connected component for $\mu_{\alpha-\alpha \lambda^{\prime}-}$ a. e. $\eta$, and so $\theta\left(\alpha^{-d} \lambda\right)>\theta\left(\alpha^{-d} \lambda^{\prime}\right) \geqq p_{c}(l)$. In view of Proposition 5.1 putting $c_{1}^{\prime}=$ $c_{1}\left(\theta\left(\alpha^{-d} \lambda\right)\right)$ and $c_{2}^{\prime}=c_{2}\left(\theta\left(\alpha^{-d} \lambda\right)\right)$, we obtain

and hence

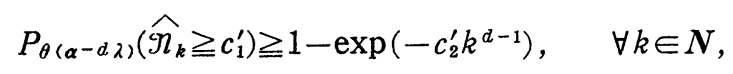

$$
\mu_{\lambda}\left(\eta \in \mathfrak{M}: \widehat{\eta}_{k}\left(\pi_{l}(\alpha \eta)\right) \geqq c_{1}^{\prime}\right) \geqq 1-\exp \left(-c_{2}^{\prime} k^{d-1}\right), \quad \forall k \in N .
$$

Noting that $n I\left[\pi_{l}(\eta)\right] \subset I\left[\pi_{n l}(n \eta)\right]$, we obtain $\widehat{\eta_{n k}}\left(\pi_{n l}(n \eta)\right) \geqq \widehat{\eta_{k}}\left(\pi_{l}(\eta)\right)$. Accordingly,

$$
\mu_{\lambda}\left(\eta \in \mathfrak{M}: \widehat{\eta_{n k}}\left(\pi_{n l}(n \alpha \eta)\right) \geqq c_{1}^{\prime}\right) \geqq 1-\exp \left(c_{2}^{\prime} k^{d-1}\right), \quad \forall k, \forall n \in N
$$

Since $q_{\eta}^{n}(x, d y) \geqq q^{n}\left[(1 / n \alpha) I\left[\pi_{n l}(n \alpha \eta)\right]\right](x, d y)$ from (5.6), we get 


$$
\mathcal{I}_{k}\left(q_{\eta}^{n}, d x\right) \geqq \mathcal{I}_{k}\left(q^{n}\left[\frac{1}{n \alpha} I\left[\pi_{n l}(n \alpha \eta)\right]\right], d x\right), \quad \forall \eta \in \mathfrak{M} .
$$

We see that $\widehat{\mathfrak{I}_{k}}\left(q^{\beta}[A], d x\right) \geqq \widehat{\mathcal{G}_{n k}}\left(q^{\beta / n}[n A], d x\right)$ by simple calculation. Then

$$
\widehat{g_{k}}\left(q^{n}\left[\frac{1}{n \alpha} I\left[\pi_{n l}(n \alpha \eta)\right]\right], d x\right) \geqq \widehat{\mathfrak{g}_{n \alpha k}}\left(q^{1 / \alpha}\left[I\left[\pi_{n l}(n \alpha \eta)\right]\right], d x\right), \quad \forall \eta \in \mathfrak{M} .
$$

From (5.1), (5.7), (5.8) and (5.9) we have

$$
\begin{aligned}
\mu_{\lambda}(\eta & \left.\in \mathfrak{M}: \widehat{g_{k}}\left(q_{\eta}^{n}, d x\right) \geqq c_{3}\left(\frac{1}{\alpha}, c_{1}^{\prime}\right)\right) \\
& \geqq \mu_{\lambda}\left(\eta \in \mathfrak{M}: \widehat{g_{k}}\left(q^{n}\left[\frac{1}{n \alpha} I\left[\pi_{n l}(n \alpha \eta)\right]\right], d x\right) \geqq c_{3}\left(\frac{1}{\alpha}, c_{1}^{\prime}\right)\right) \\
& \geqq \mu_{\lambda}\left(\eta \in \mathfrak{M}: \widehat{g_{n \alpha k}}\left(q^{1 / \alpha}\left[I\left[\pi_{n l}(n \alpha \eta)\right]\right], d x\right) \geqq c_{3}\left(\frac{1}{\alpha}, c_{1}^{\prime}\right)\right) \\
& \geqq \mu_{\lambda}\left(\eta \in \mathfrak{M}: \widehat{\Re_{n \alpha k}}\left(\pi_{n l}(n \alpha \eta)\right) \geqq c_{1}^{\prime}\right) \\
& \geqq 1-\exp \left(-c_{2}^{\prime} k^{d-1}\right) .
\end{aligned}
$$

Since $c_{3}\left(1 / \alpha, c_{1}^{\prime}\right)>0$ from Lemma 5.1 , Lemma 4.2 is obtained by Borel-Cantelli's Lemma.

Acknowledgement. The author would like to thank Professor H. Osada for his valuable suggestion.

\section{REFERENCES}

[1] BAss, R.F. AND Hsu, P., Some potential theory for reflecting Brownian motion in Hölder and Lipschitz domains, Ann. Probab., 19 (1991), 486-508.

[2] BhatTachaRYa, R., A central limit theorem for diffusions with periodic coefficients, Ann. Prob., 13 (1985), 385-396.

[3] Burton, R.M. and Keane, M., Density and uniqueness in percolation, Comm. Math. Phys., 121 (1989), 501-505.

[4] Chayes, J. T. And Chayes, L., Bulk transport properties and exponent inequalities for random resistor and flow networks, Comm. Math. Phys., 105 (1986), 133-152.

[5] De Masi, A., Ferrari, P. A., Goldstein, S. and Wick, D. W., An invariance principle for reversible Markov processes. Applications to random motions in random environments, J. Statist. Phys., 55 (1989), 787-855.

[6] Doyle, P.G. ANd SNell, J.L., Random Walks and Electrical Networks, Washington DC, Math. Assoc. Amer., 1984.

[7] Fukushima, M., On the boundary conditions for multidimensional Brownian motions with symmetric resolvent densities, J. Math. Soc. Japan, 21 (1969), 58-93.

[8] Fukushima, M., Dirichlet Form and Markov Processes, North-Holland/Kodansha, 1980.

[9] Grimmett, G.R., Percolation, Springer-Verlag, 1989. 
[10] Grimmett, G.R. And Kesten, H., First passage percolation, network flows and electrical resistances, Z. Wahrsch. Verw. Geb., 66 (1984), 335-366.

[11] Grimmett, G.R. And Marstrand, J.M., The supercritical phase of percolation is well behaved, Proc. Roy. Soc. Lond. Ser. A, 430 (1990), 439-457.

[12] Kipnis, C. AND Varadhan, S.R.S., Central limit theorems for additive functionals of reversible Markov processes and applications to simple exclusions, Comm. Math. Phys., 104 (1986), 1-19.

[13] Lyons, T. AND Zheng, W., A crossing estimate for the canonical process on a Dirichlet space and tightness result, Asterisque, 157-158 (1988), 249-271.

[14] Ocнi, Y., Limit theorems for a class of diffusion processes, Stochastics, 15 (1985), 251-269.

[15] Osada, H., Homogenization of reflecting barrier Brownian motions, Asymptotic Problems in Probability Theory: Stochastic Models and Diffusions on Fractal (eds. K.D. Elworthy \& N. Ikeda), Pitman Research Notes in Math., 283, Longman, 1993, 59-74.

[16] Roy, R., The Russo-Welsh theorem and the equality of critical densities and the dual critical densities for continuum percolation on $\boldsymbol{R}^{d}$, Ann. Probab., 18 (1990), 1563-1575.

[17] Stein, E. M., Singular Integrals and Differentiability Properties of Functions, Princeton University Press, 1970.

[18] TAKEDA, M., On a martingale method for symmetric diffusion processes and its application, Osaka J. Math., 26 (1989), 605-623,

[19] TANemura, H., Central limit theorem for a random walk with random obstacles in $\boldsymbol{R}^{d}$, Ann. Probab., 21 (1993), 936-960.

[20] TANemura, H., Behavior of the supercritical phase of a continuum percolation model on $\boldsymbol{R}^{d}$, J. Appl. Prob., 30 (1993), 382-396.

[21] Trotter, H.F., Approximation of semigroups of operators, Pacific J. Math., 8 (1958), 887-919.

Department of Mathematics

FACULTY OF SCIENCE

Chiba University

ChIBA, 263 JAPAN 